\title{
Inhibition by long-chain acyl-CoAs of glucose 6-phosphate metabolism in plastids isolated from developing embryos of oilseed rape (Brassica napus L.)
}

\author{
Philip E. JOHNSON ${ }^{1}$, Simon R. FOX, Matthew J. HILLS and Stephen RAWSTHORNE \\ John Innes Centre, Norwich Research Park, Colney, Norwich, Norfolk NR4 7UH, U.K.
}

\begin{abstract}
The effects of long-chain acyl-CoA (lcACoA) esters (both added exogenously and synthesized de novo) and acyl-CoA binding protein (ACBP) on plastidial glucose 6-phosphate (Glc6P) and pyruvate metabolism were examined using isolated plastids. The binding of lcACoA esters by ACBP stimulated the utilization of Glc6P for fatty acid synthesis, starch synthesis and reductant supply via the oxidative pentose phosphate (OPP) pathway. Stimulation occurred at low $(1-10 \mu \mathrm{M})$ concentrations of ACBP. Pyruvate-dependent fatty acid synthesis was not directly affected by ACBP. However, addition of ACBP did increase the Glc6P-
\end{abstract}

dependent stimulation of pyruvate utilization mediated through the OPP pathway. On the basis of these experiments, we conclude that lcACoA esters may inhibit Glc6P uptake into plastids, and that this inhibition is relieved by ACBP. We also suggest that utilization of other substrates for fatty acid synthesis may be affected by lcACoA/ACBP via their effects on the OPP pathway.

Key words: acyl-CoA binding protein, acyl-CoA esters, fatty acid synthesis.

\section{INTRODUCTION}

Fatty acid synthesis in plants occurs entirely in the plastid. These fatty acids are then exported into the cytosol for modification and subsequent utilization by the endoplasmic reticulum-localized enzymes of the Kennedy pathway for storage and membrane lipid synthesis. The substrates for the acyltransferase enzymes of the Kennedy pathway are long-chain acyl-CoA (lcACoA) esters [1], and fatty acids must therefore be activated by acyl-CoA synthetase prior to their further use in lipid synthesis.

In animals and yeast, there is much evidence to suggest that fatty acid synthesis is regulated by lcACoA esters. In particular, it has been demonstrated that mammalian acetyl-CoA carboxylase (ACCase; the first committed step of fatty acid synthesis) is inhibited by sub-micromolar concentrations of lcACoA esters and fatty acids [2]. Kamiryo et al. [3] showed that inhibition of fatty acid synthesis in yeast by exogenously added fatty acids is dependent upon their conversion into lcACoA esters by acylCoA synthetase. It is thought that lcACoA esters regulate their own synthesis by inhibiting the production of malonyl-CoA by ACCase and thus decreasing the flux of acetyl-CoA towards fatty acids.

The discovery of acyl-CoA binding proteins (ACBPs) provides a possible mechanism by which the cytosolic lcACoA esters pool may be maintained. ACBP is a small (approx. $10 \mathrm{kDa}$ ) cytosolic protein which binds lcACoA esters and has no other known function [4]. ACBPs have been shown to protect the lcACoA pool from hydrolysis by acyl-CoA thioesterase activity [5] and may also be involved in the protection of cell membranes from the detergent effects of lcACoAs.

lcACoA esters are important signalling and regulatory molecules [4]. In many cases where the inhibition of an enzyme or process by lcACoAs has been observed, the inhibition is reversible by the addition of $\mathrm{ACBP}$ in an equal or excess concentration. For example, inhibition of mammalian ACCase by $\mathrm{lcACoA}$ esters is reversed by ACBP [6] and it is therefore thought to be the ratio of $1 \mathrm{cACoA}$ esters to ACBP that is important in determining the level of inhibition of ACCase.

In plants, the enzymology of plant lipid synthesis is relatively well understood. However, much less is known about the regulation of fatty acid synthesis. In leaves, fatty acid synthesis may be regulated by light via changes in the activity of ACCase [7], possibly via redox potential [8] or phosphorylation [9]. In addition, it has been demonstrated that exogenously added fatty acids are inhibitory to de novo fatty acid synthesis in tobacco leaf suspension cultures, indicating that fatty acids may regulate their own synthesis [10], again most probably by affecting the activity of ACCase. In the latter experiments it is not known if inhibition of fatty acid synthesis occurred by fatty acids themselves or after their conversion into acyl-CoA esters.

The lcACoA ester pool is thought to be located in the cytosol and all known soluble ACBPs are cytosolic [4]. In order for lcACoA esters to act as regulators of fatty acid synthesis in plants, they must exert control over fatty acid synthesis from outside the plastid. Studies of fatty acid synthesis in plants have been performed extensively using isolated plastids, a system which allows free manipulation of the external environment of the plastid. We therefore used isolated plastids to study the effect of lcACoA esters and ACBP on plastid metabolism. Plastids isolated from oilseed rape (Brassica napus L.) have been extensively characterized and they utilize glucose 6-phosphate (Glc6P) and pyruvate most readily as substrates for fatty acid synthesis [11]. Glc6P is also utilized by these plastids for starch synthesis and as a substrate for reductant supply via the oxidative pentose phosphate (OPP) pathway [12]. In this study

Abbreviations used: ACBP, acyl-CoA binding protein; Glc6P, glucose 6-phosphate; OPP, oxidative pentose phosphate; IcACoA, Iong-chain acylCoA; ACCase, acetyl-CoA carboxylase; PIM, plastid isolation medium; GAPdH, glyceraldehyde-3-phosphate dehydrogenase.

1 To whom correspondence should be addressed (e-mail p.johnson@bbsrc.ac.uk). 
we therefore utilized Glc6P and pyruvate as substrates for plastid metabolism.

\section{MATERIALS AND METHODS}

\section{Enzymes and special reagents}

All chemicals, substrates and coupling enzymes were purchased from either Sigma Chemical Co. (Poole, Dorset, U.K.) or Boehringer (Lewes, Sussex, U.K.) unless stated otherwise. Radiolabelled isotopes were purchased from Amersham International (Amersham, Bucks., U.K.).

\section{Overexpression and purification of ACBP}

B. napus ACBP was over-expressed in Escherichia coli (BL21) and purified as described in Brown et al. [13].

\section{Isolation of plastids}

Plastids were isolated from developing embryos of B. napus cv Topas (approx 30-33 days after anthesis) essentially by the method of Kang and Rawsthorne [11] with the following modifications. BSA was omitted from plastid isolation medium (PIM) at all stages of the procedure. After the generation of the crude plastid pellet, plastids were resuspended in $5 \mathrm{ml}$ of PIM and gently layered on top of $5 \mathrm{ml}$ of PIM containing $35 \%(\mathrm{v} / \mathrm{v})$ Percoll (Sigma) and then centrifuged at $1000 \mathrm{~g}$ for $8 \mathrm{~min}$. The resultant pellet was washed as described in Kang and Rawsthorne [11]. Leaf chloroplasts were prepared by the method of Eastmond and Rawsthorne [14].

\section{Plastid feeding experiments}

Plastids were supplied with radiolabelled precursors for starch synthesis, fatty acid synthesis and the OPP pathway exactly as described by Kang and Rawsthorne [12] except that the total volume of the incubations was $166 \mu 1$.

\section{Extraction of radiolabelled lipids}

Total saponifiable lipids were extracted and quantified as described in Kang and Rawsthorne [11]. Analysis of lipid classes was achieved by extraction with chloroform/methanol followed by TLC. Plastid incubations were stopped by the addition of $0.8 \mathrm{ml}$ of chloroform/methanol $(1: 1, \mathrm{v} / \mathrm{v})$ and $0.3 \mathrm{ml}$ of $0.1 \%$ acetic acid $/ 10 \%(\mathrm{w} / \mathrm{v}) \mathrm{KCl}$. The resultant biphase was cleared by centrifugation in a microcentrifuge at $10000 \mathrm{~g}$ for $5 \mathrm{~min}$ and the lower (organic) phase removed, dried under nitrogen and resuspended in $50 \mu \mathrm{l}$ of chloroform/methanol $(1: 1, \mathrm{v} / \mathrm{v})$. The supernatant was retained for extraction of lcACoA esters (see below). Lipid samples in chloroform/methanol $(1: 1, \mathrm{v} / \mathrm{v})$ were applied to silica gel TLC plates (Analtech, Newark, DE, U.S.A.) Separation of lipid classes was achieved using the following solvent systems: chloroform/methanol/acetic acid $/ \mathrm{H}_{2} \mathrm{O}$ $(50: 30: 8: 4$, by vol.) for separation of polar lipids and hexane/ diethyl ether/acetic acid (70:30:1, by vol.) for separation of neutral lipids. Relevant standards were applied to at least one lane of every plate.

Long-chain esters were extracted from the aqueous phase of the extraction procedure described above. Aqueous phases were dried under $\mathrm{N}_{2}$ and resuspended in $0.4 \mathrm{ml}$ of butan-1-ol. After the addition of $0.6 \mathrm{ml}$ of water the resultant biphase was clarified by centrifugation at $10000 \mathrm{~g}$ in a microcentrifuge. The upper (butanol) phase containing approximately $75 \%$ of the lcACoA esters was removed and dried under nitrogen. Samples were then resuspended in $50 \mu \mathrm{l}$ of butan-1-ol/acetic acid/water $(50: 20: 30$, by vol.) prior to application to silica gel TLC plates. Acyl-CoA esters were resolved in butan-1-ol/acetic acid/water $(50: 20: 30$, by vol.).

After overnight drying, standards were visualized by iodine spraying and radiolabelled lipid classes visualized by autoradiography. Quantification of radiolabelled bands was achieved by scraping relevant areas from the plate and liquid scintillation counting.

\section{Analysis of fatty acids by HPLC}

Total-saponified fatty acid samples were prepared as described above. Fatty acid samples and standards were derivatized to nbromophenacyl esters as described by Durst et al. [15]. Resolution of n-bromophenacyl esters was achieved using an Ultratechsphere $5 \mu \mathrm{m}$ particle size ODS C18 reverse-phase column (HPLC Technology Company Ltd., Macclesfield, Cheshire, U.K.) and a Gilson HPLC system. Column flow was $1 \mathrm{ml} \cdot \mathrm{min}^{-1}$. The n-bromophenacyl ester standards were detected at $A_{210}$. Radiolabelled fatty acid derivatives were detected by liquid scintillation counting of $1 \mathrm{ml}$ fractions.

\section{Determination of starch synthesis and the OPP pathway}

Utilization of $\left[1-{ }^{14} \mathrm{C}\right]$ Glc6P for starch synthesis or via the OPP pathway was measured as described by Kang and Rawsthorne [12].

\section{Localization of the products of de novo fatty acid synthesis}

The localization of de novo synthesized acyl chains was adapted from methods for the determination of metabolite uptake into plastids [16]. Five minutes from the incubation stop time, incubations were layered on top of $80 \mu \mathrm{l}$ of AR100/AR200 (2:1, v/v) silicone oil (Wacker Chemicals Ltd., Walton-on-Thames, Surrey, U.K.) which was layered on top of $40 \mu 1$ of 0.7 M sucrose in a $400 \mu \mathrm{l}$ Eppendorf reaction vessel. At the incubation stop time, tubes containing incubations were centrifuged at $15000 \mathrm{~g}$ for $10 \mathrm{~s}$. Intact plastids were pelleted in the sucrose, while soluble material remained above the silicone oil. Tubes were then frozen in liquid $\mathrm{N}_{2}$ and cut into fractions containing the sucrose cushion and the original incubation. Each of these fractions was made up to $165 \mu \mathrm{l}$ with deionized water and the extraction of total fatty acids carried out as described previously.

\section{Measuring Glc6P uptake}

Plastids in PIM were pre-incubated in a total volume of $155 \mu 1$ with a final concentration of $1.2 \mu \mathrm{M}$ oleoyl-CoA or with no addition (for controls) for $2 \mathrm{~min}$ at $25^{\circ} \mathrm{C}$. After $90 \mathrm{~s}$ of this preincubation, the plastids were drawn up into the same pipette tip. Plastids were mixed (30 s later) with $10 \mu \mathrm{l}$ of $\left[1-{ }^{14} \mathrm{C}\right]$ Glc6P $(5.9$ $\mathrm{kBq}$ ) to a final concentration of $50 \mu \mathrm{M}$. Upon mixing with the isotope, plastids were immediately removed and maintained in the pipette tip for $30 \mathrm{~s}$. Plastids were then transferred into a $400 \mu \mathrm{l}$ Eppendorf reaction vessel containing silicone oil and sucrose as described above. The vessel containing the silicone oil and plastids was centrifuged at $15000 \mathrm{~g}$ for $20 \mathrm{~s}$. After centrifugation, a razor blade was used to cut through the centre of the oil layer. The plastids (in the sucrose layer) were transferred directly into $10 \mathrm{ml}$ of scintillation fluid. The lower half of the silicone oil tube was then washed twice with water and once with methanol before adding the cut-off end of the tube and the pipette tip to the scintillation fluid prior to scintillation counting. 


\section{RESULTS}

Although it has previously been demonstrated that Glc6P is an effective substrate for fatty acid synthesis in oilseed rape $(B$. napus) embryo plastids [11], the composition of fatty acids synthesized by these isolated plastids in terms of lipid class and fatty acid type are not known. We therefore analysed the products of plastidial fatty acid synthesis by TLC to determine the class of lipids synthesized, and by n-bromophenacyl derivatization and HPLC to determine the type of fatty acids produced. The major radiolabelled fatty acid product detectable after incubation of plastids with Glc6P was $\mathrm{C}_{18: 1}$ (oleic acid), with both $\mathrm{C}_{16: 1}$ (palmitic acid) and $\mathrm{C}_{18: 0}$ (stearic acid) also detectable (Figure 1a). These observations are consistent with the substrate specificity of known B. napus acyl-carrier protein thioesterases [17] and the abundance of oleic acid in seed storage oil at desiccation [18]. The predominant lipid class synthesized from Glc6P was lcACoA esters, with lower but detectable quantities of diacylglycerol, triacylglycerol and polar lipids also synthesized (Figure 1b). Fatty acid metabolism in these plastids therefore included both functional acyl-carrier protein thioesterase and acyl-CoA synthetase activity. As plastids synthesized lcACoA esters, the effect of their binding by ACBP after de novo synthesis could therefore be examined.

The presence of $10 \mu \mathrm{M}$ ACBP or $152 \mu \mathrm{M}$ BSA, a protein which binds both fatty acids and lcACoA esters [19,20], stimulated fatty acid synthesis by 3-4-fold over incubations containing a control protein, cytochrome $c(10 \mu \mathrm{M})$ or no added protein (Figure 2a). Cytochrome $c$ was used as a control as it is of a molecular mass similar to ACBP (approx. $10 \mathrm{kDa}$ ) and has no known lcACoA-ester-binding capacity. The stimulation of Glc6P-dependent fatty acid synthesis by ACBP and BSA still occurred when plastids were incubated with substrate for $40 \mathrm{~min}$ prior to the addition of ACBP (Figure 2b). The rate of in-

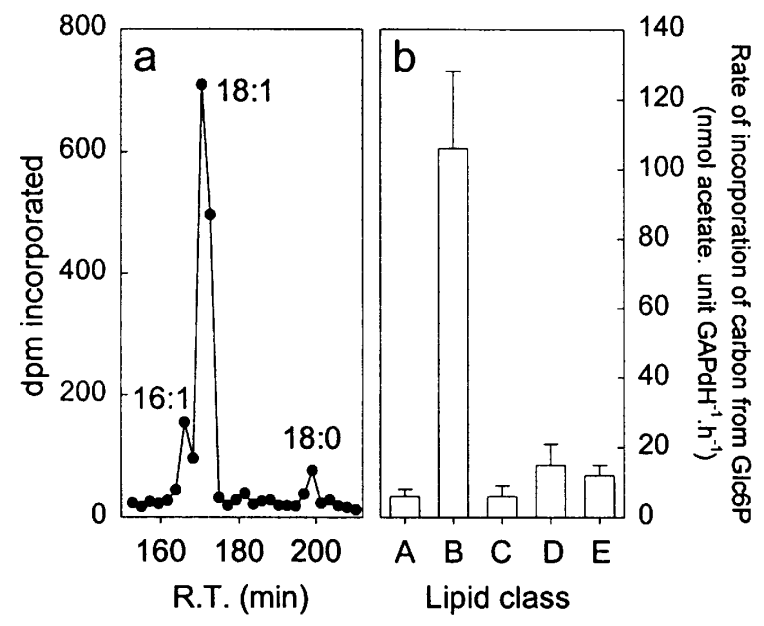

Figure 1 Analysis of the end-products of fatty acid synthesis by isolated plastids

Plastids were incubated with $1 \mathrm{mM}\left[1-{ }^{14} \mathrm{C}\right] \mathrm{Glc} 6 \mathrm{P}$ in the presence of $152 \mu \mathrm{M} \mathrm{BSA}$. After $1 \mathrm{~h}$, incubations were stopped and lipids extracted for the determination of fatty acid type (a) or lipid class (b) as described in the Materials and methods section. In both cases, fatty acid type or lipid class was identified by comparison with known authentic standards. (a) Fatty acid type is shown on the Figure (16:1, palmitic acid; 18:1, oleic acid; 18:0, stearic acid). R. T., retention time. The experiment was performed twice on individual plastid preparations and a representative chromatogram is shown. (b) Lipid classes: A, free fatty acid; B, IcACoA esters; C, triacylglycerol; D, polar lipids; E, diacylglycerol. Results are the mean \pm S.E.M. for experiments performed on three individual plastid preparations.

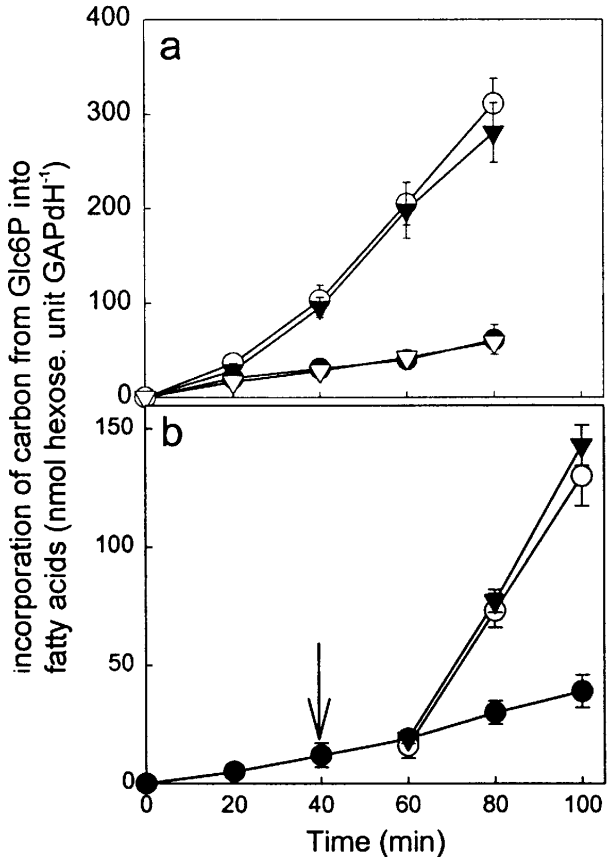

Figure 2 Time course of fatty acid synthesis from GIc6P

(a) Plastids were incubated with $1 \mathrm{mM}\left[1-{ }^{14} \mathrm{C}\right] \mathrm{Glc} 6 \mathrm{P}$ in the absence of additional added protein ) or in the presence of $152 \mu \mathrm{M}$ BSA (O), $10 \mu \mathrm{M}$ ACBP $(\boldsymbol{\nabla})$ or $10 \mu \mathrm{M}$ cytochrome $c$ $\nabla)$. Total fatty acids were extracted and quantified as described in the Materials and methods section. (b) Plastids were incubated with $\left[1-{ }^{14} \mathrm{C}\right] \mathrm{Glc} 6 \mathrm{P}$ for 40 min prior to the addition of $10 \mu \mathrm{M}$ ACBP $(\bigcirc)$ or $152 \mu \mathrm{M}$ BSA $(\boldsymbol{\nabla})$. The time of addition is shown with an arrow. Other incubations were continued in the absence of added protein $(\boldsymbol{)})$. Results are the mean \pm S.E.M. for experiments performed on three separate plastid preparations.

corporation of carbon from Glc6P into fatty acids that was attained after delayed protein addition was equal to that attained where either of the added proteins was present from the start of the incubation. It can therefore be concluded that the lower rates of fatty acid synthesis in the absence of BSA or ACBP were not due to irreversible damage to the plastid in the absence of either protein. In addition, the intactness of plastids after $30 \mathrm{~min}$ incubation with $10 \mu \mathrm{M}$ ACBP, $152 \mu \mathrm{M}$ BSA or $20 \mu \mathrm{M}$ oleoyl$\mathrm{CoA}$ was estimated by measurement of $\mathrm{NADP}^{+}$-glyceraldehyde3-phosphate dehydrogenase (GAPdH) latency (results not shown). None of these incubation conditions resulted in a significant decrease in $\mathrm{NADP}^{+}-\mathrm{GAPdH}$ latency $(P>0.05)$, suggesting that plastid intactness was unaffected by free lcACoA esters in the incubation medium.

The ACBP-mediated stimulation of Glc6P-dependent fatty acid synthesis was strongly dependent on the concentration of ACBP (Figure 3). Half-maximal stimulation of fatty acid synthesis occurred at approx. 3.5 $\mu \mathrm{M}$ ACBP. To examine whether the stimulation of Glc6P metabolism by ACBP was specific to fatty acid synthesis, the effect of ACBP addition on Glc6Pdependent starch synthesis by the plastids was also studied. Surprisingly, ACBP stimulated the incorporation of carbon from Glc6P into starch by approx. 2.5-fold in comparison with no protein controls (Figure 3), with half-maximum stimulation occurring at $2.7 \mu \mathrm{M}$ ACBP. The only known function of ACBP is to bind lcACoA esters, and ACBP does not bind either free fatty acids or CoA [21]. The stimulation of both fatty acid and starch synthesis from Glc6P by ACBP provides indirect evidence 


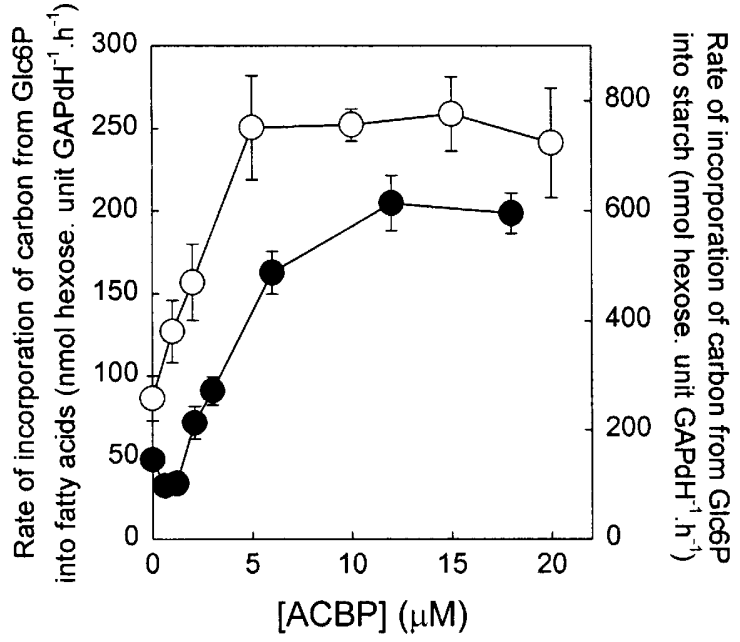

Figure 3 The effect of ACBP concentration on the rates of starch and fatty acid synthesis from Glc6P

Plastids were incubated with $1 \mathrm{mM}\left[1-{ }^{14} \mathrm{C}\right] \mathrm{Gl} 6 \mathrm{P}$ in the presence of different concentrations of ACBP for $1 \mathrm{~h}$. Radioactively labelled fatty acids $(\mathbf{O})$ or starch $(\bigcirc)$ were then extracted and quantified as described in the Materials and methods section. Results are the mean \pm S.E.M. for experiments performed on three separate plastid preparations.

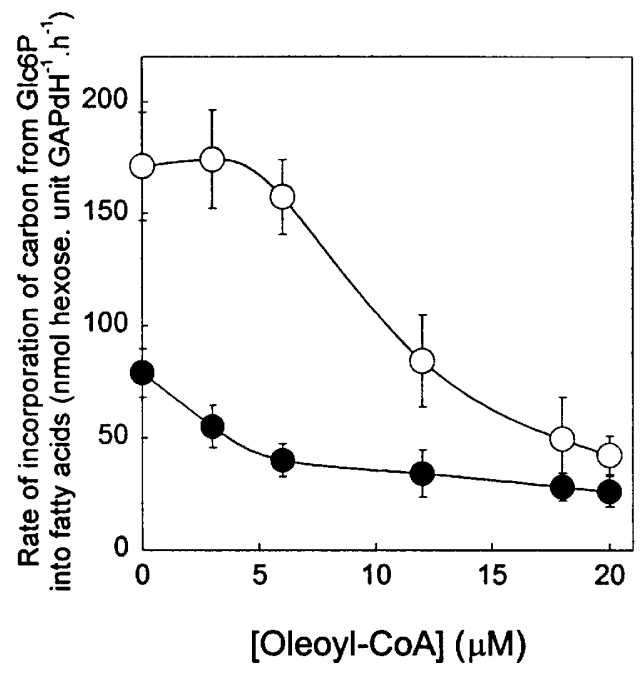

Figure 4 The effect of oleoyl-CoA concentration on fatty acid synthesis from GIc6P

Plastids were incubated with $1 \mathrm{mM}\left[1-{ }^{14} \mathrm{C}\right] \mathrm{Glc} 6 \mathrm{P}$ in the presence of different concentrations of oleoyl-CoA, and in the absence $(\mathbf{O})$ or presence $(\mathrm{O})$ of $5 \mu \mathrm{M}$ ACBP. Results are the mean \pm S.E.M. for experiments performed on three separate plastid preparations.

that lcACoA esters can inhibit both of these plastidial biosynthetic pathways. In order to study this further, lcACoA esters were added to plastid incubations containing either no additional protein or $5 \mu \mathrm{M}$ ACBP (Figure 4). In the absence of ACBP, oleoyl-CoA concentrations up to $20 \mu \mathrm{M}$ decreased the rate of fatty acid synthesis, with $20 \mu \mathrm{M}$ oleoyl-CoA decreasing the rate of fatty acid synthesis to $39 \%$ of the control rate. In contrast, in the presence of $5 \mu \mathrm{M}$ ACBP, oleoyl-CoA concentrations up to $5 \mu \mathrm{M}$ had no significant effect on the rate of fatty acid synthesis. Increasing the oleoyl-CoA concentration further, however, inhibited fatty acid synthesis, with $20 \mu \mathrm{M}$ oleoyl-CoA reducing the

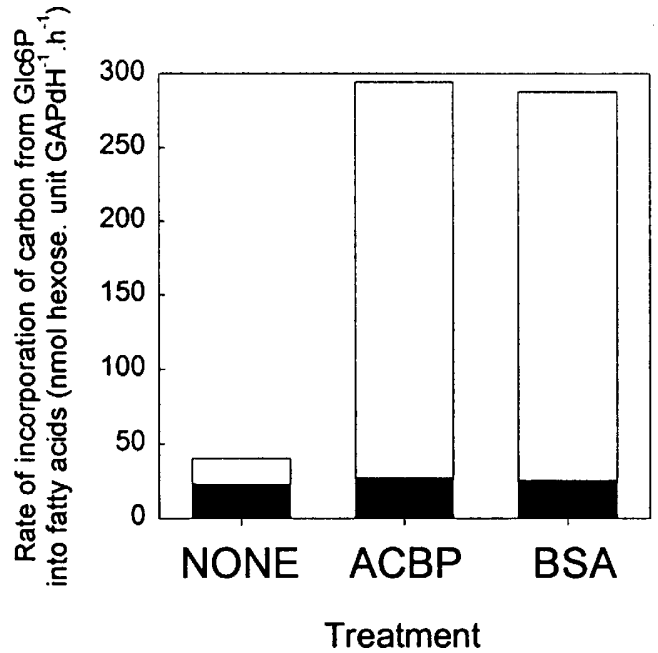

Figure 5 Localization of the products of fatty acid synthesis by isolated plastids

Plastids were incubated with $1 \mathrm{mM}\left[1-{ }^{14} \mathrm{C}\right] \mathrm{Glc} 6 \mathrm{P}$ in the absence of additional protein, the presence of $10 \mu \mathrm{M}$ ACBP or the presence of $152 \mu \mathrm{M}$ BSA for $1 \mathrm{~h}$. Plastid incubations were then centrifuged through silicone oil and fatty acids were extracted from the remaining incubation medium. Filled bars, fatty acids present in the plastid fraction (internal fatty acids); open bars, fatty acids present in the incubation medium (external fatty acids). Results are means for experiments performed on three separate plastid preparations. The S.E.M. was within $12 \%$ of the mean in each case.

rate to $26 \%$ of the control. The inhibition by oleoyl-CoA only occurred when its concentration was in molar excess to that of ACBP. The recombinantly expressed ACBP of B. napus binds lcACoA esters in a molar ratio of $1: 1$ [13]. It is therefore very likely that only free lcACoA esters inhibit fatty acid synthesis in plastids, similar to the inhibition of mammalian ACCase by lcACoA [6].

These experiments describe the first observation of the inhibition of plastidial fatty acid synthesis by their cytosolic end-product, lcACoA esters. Because of the cytosolic location of both lcACoA esters and ACBP, it seems likely that the site of their effect is either cytosolic or on the plastid envelope membrane. In the current experiments, isolated plastids were enriched in $\mathrm{NADP}^{+}-\mathrm{GAPdH}$ (a plastid marker enzyme) relative to alcohol dehydrogenase (a cytosolic marker enzyme) by approximately 56-fold (results not shown). In addition, Glc6P has been shown to enter the plastid directly via a specific metabolite transporter on the plastid inner envelope prior to fatty acid synthesis [14]. It is therefore extremely unlikely that the observed effects of lcACoA esters and ACBP on plastid metabolism are due to an effect mediated by extraplastidial metabolism of Glc6P. The location of lcACoA esters synthesized during plastid incubations was studied by separating plastids from the external incubation mixture using silicone oil centrifugation. This technique has been extensively used to measure metabolite uptake into plastids [16]. In these experiments, the location of the total radiolabelled acyl-chain products and not of the lcACoA esters was determined. This was due to difficulty in extraction of lcACoA esters from the sucrose cushion after centrifugation of plastids through silicone oil. In the absence of added protein the rate of fatty acid synthesis was low and the products were evenly distributed between the plastids and the medium (Figure 5). Under incubation conditions that allowed high rates of fatty acid synthesis (in the presence of either BSA or ACBP), ${ }^{14} \mathrm{C}$-labelled acyl 


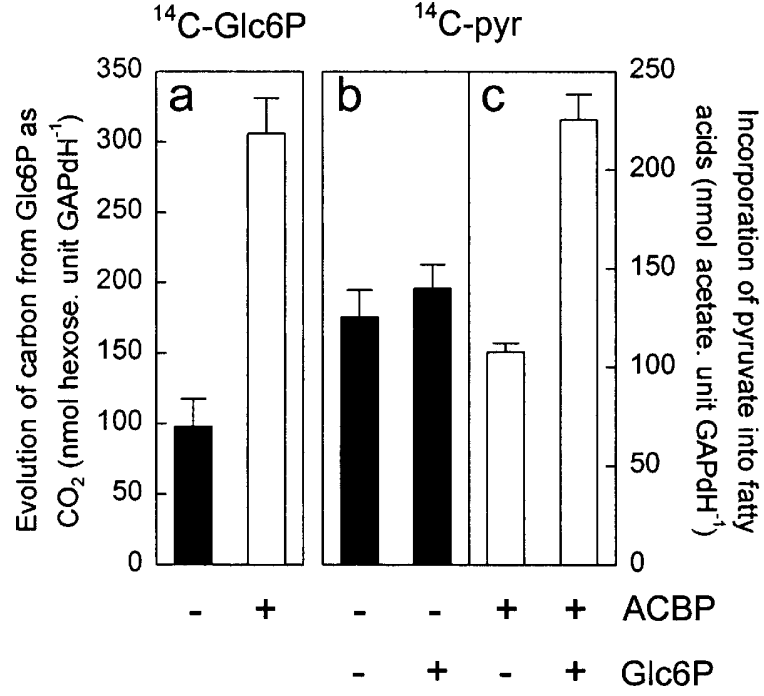

Figure 6 The effect of ACBP on the supply of GIc6P to the OPP pathway

(a) Direct measurement of the OPP pathway. Plastids were incubated with $1 \mathrm{mM}\left[1-{ }^{14} \mathrm{C}\right] \mathrm{Glc} 6 \mathrm{P}$ in the absence of additional protein (filled bars) or presence of $10 \mu \mathrm{M}$ ACBP (open bars) for $1 \mathrm{~h}$. The evolution of ${ }^{14} \mathrm{CO}_{2}$ was used to measure the activity of the OPP pathway. (b and $\mathbf{c}$ ) Indirect measurement of the OPP pathway through stimulation of the incorporation of carbon from pyruvate into fatty acids by Glc6P metabolism. Plastids were incubated with $\left[2-{ }^{14} \mathrm{C}\right]$ pyruvate $\left({ }^{14} \mathrm{C}\right.$-pyr) in the absence or presence of unlabelled $1 \mathrm{mM}$ Glc6P. Bar shading denoting treatments are as described in (a). Results are the mean \pm S.E.M. for experiments performed on three separate plastid preparations.

products, which were predominantly lcACoA esters (Figure 1b), were present almost exclusively outside the plastid. lcACoA esters are thought to be predominantly cytosolic in vivo. These data provide further evidence that the site of action of lcACoA esters and ACBP is outside the plastid.

There are three possible common sites of inhibitory action by lcAcCoAs that can account for the observed reduction in the rates of Glc6P-dependent starch and fatty acid synthesis. First, the import of Glc6P itself as this is the common precursor. Second, the import of ATP since both biosynthetic reactions are dependent upon ATP supply in vitro $[11,12]$. Precedent for these two possible sites of action comes from reports that Glc6P transport into mammalian microsomes [22] and ATP transport into mammalian mitochondria $[23,24]$ are inhibited in vitro by low micromolar concentrations of 1cACoA esters. A third possibility is that an as yet undefined feedback effect, caused by the inhibition of acyl chain export, affects plastidial starch and fatty acid synthesis. To examine whether the first possibility is correct, plastids were supplied with an alternative substrate, pyruvate, in the presence or absence of ACBP. In contrast with the results where Glc6P was supplied, the rate of pyruvatedependent fatty acid synthesis was unaffected by ACBP (Figure 6 b). Similarly, utilization of pyruvate or acetate for fatty acid synthesis by chloroplasts derived from leaves of oilseed rape seedlings was unaffected by ACBP or BSA $(P>0.05$; results not shown). These observations reveal that the synthesis of lcACoAs per se is not the cause of inhibition. It can also be concluded that inhibition of ATP uptake is not a site of inhibition since both pyruvate- and Glc6P-dependent fatty acid synthesis are ATP dependent [14] and should have been affected similarly by the accumulation of lcACoAs in the absence of exogenous ACBP. In order to examine the effect of oleoyl-CoA on Glc6P transport into the plastid, short-term metabolite-transport assays were performed. These assays represent a direct measurement of the transport of metabolites into plastids [16]. The presence of $1.2 \mu \mathrm{M}$ oleoyl-CoA inhibited the uptake of Glc6P into plastids by $75-80 \%$ compared with control experiments $(P<0.05$; results not shown).

If the effect of lcACoAs was confined to Glc6P uptake then Glc6P-dependent processes in the plastid other than starch or fatty acid synthesis should be similarly inhibited by lcACoAs. Glc6P is a substrate for the OPP pathway in plastids of oilseed rape embryos [12]. It is argued that Glc6P stimulates incorporation of carbon from pyruvate into fatty acids through the generation of reducing equivalents by the OPP pathway [12,25] in order to satisfy the requirement for $\mathrm{NAD}(\mathrm{P}) \mathrm{H}$ of the plastidial fatty acid synthetase complex [26]. The activity of the plastidial OPP pathway in incubations containing Glc6P as the sole substrate was increased 3 -fold by the presence of ACBP (Figure 6a) providing further support for the hypothesis that 1cAcCoAs inhibit Glc6P uptake. Indeed, when plastids were supplied with $\left[2-{ }^{14} \mathrm{C}\right]$ pyruvate and unlabelled Glc6P there was a greater than 2-fold increase in the rate of pyruvate-dependent fatty acid synthesis when ACBP was present (Figure 6c). Under the same incubation conditions but in the absence of ACBP there was no stimulation of pyruvate-dependent fatty acid synthesis by Glc6P (Figure 6b).

\section{DISCUSSION}

The observations that synthesis of lcACoAs in the absence of a binding protein leads to (i) a reduction in Glc6P-dependent starch and fatty acid synthesis, and (ii) a reduction in the Glc6Pdependent stimulation of incorporation of carbon from pyruvate into fatty acids, are most simply explained by inhibition of the uptake of Glc6P across the plastid envelope. This is the only point in plastid metabolism that is common to all of these processes. The experiments described here also clearly show that the inhibition is relieved by the presence of ACBP. In all tissues studied to date, the relative concentration of lcACoA esters and that of ACBP are close to unity [4]. The inhibition of plastidial fatty acid synthesis by unbound lcACoAs provides a possible short-term mechanism by which the parity of the lcACoA and ACBP concentrations are maintained in plants. Inhibition of the carbon supply to the OPP pathway will restrict the supply of reductant to fatty acid synthetase and could therefore affect fatty acid synthesis and the production of acyl-CoAs irrespective of the cytosolic carbon source. We are currently measuring in detail the inhibitory effects of acyl-CoAs on the transporters of $B$. napus plastids with a focus on the previously characterized Glc6P transporter [14].

Mr lan Hagon and Mrs Ruth Pothecary are thanked for horticultural assistance. This work was supported by the Biotechnology and Biological Sciences Research Council (BBSRC) through its Competitive Strategic Grant to the John Innes Centre. P. E. J. was supported by a John Innes Foundation Studentship and S. R. F. was supported by a grant from the BBSRC through their Resource Allocation and Stress in Plants Initiative.

\section{REFERENCES}

1 Ohlrogge, J. and Browse, J. (1995) Plant Cell 7, 957-970

2 Ogiwara, H., Tanabe, T., Nikiwara, J.-I. and Numa, S. (1978) Eur. J. Biochem. 89 33-41

3 Kamiryo, T., Parthasarathy, S. and Numa, S. (1976) Proc. Natl. Acad. Sci. U.S.A. 73, 386-390

4 Faergeman, N. J. and Knudsen, J. (1997) Biochem. J. 323, 1-12

5 Engeseth, N. J., Pacovsky, R. S., Newman, T. and Ohlrogge, J. B. (1996) Arch. Biochem. Biophys. 331, 55-62 
6 Rasmussen, J. T., Rosendal, J. and Knudsen, J. (1993) Biochem. J. 292, 907-913

7 Post-Beitenmiller, D., Roughan, G. and Ohlrogge, J. B. (1992) Plant Physiol. 100, 923-930

8 Kozaki, A and Sasaki, Y (1999) Biochem. J. 339, 541-546

9 Savage, L. J. and Ohlrogge, J. B. (1999) Plant J. 18, 521-527

10 Shintani, D. and Ohlrogge, J. (1995) Plant J. 7, 577-587

11 Kang, F. and Rawsthorne, S. (1994) Plant J. 6, 795-805

12 Kang, F. and Rawsthorne, S. (1996) Planta 199, 321-327

13 Brown, A. P., Johnson, P., Rawsthorne, S. and Hills, M. J. (1998) Plant Physiol. Biochem. 36, 629-635

14 Eastmond, P. J. and Rawsthorne, S. (1998) J. Exp. Bot. 49, 1105-1111

15 Durst, H. D., Milano, M., Kikta, E. J., Connelly, S. A. and Grushka, E. (1975) Anal. Chem. 47, 1797-1801

16 Heldt, H. W. (1980) Methods Enzymol. 69, 604-613

17 Hellyer, A., Leadlay, P. F. and Slabas, A. R. (1992) Plant Mol. Biol. 20, 763-780
18 Murphy, D. J. and Cummins, I. (1988) J. Plant Physiol. 135, 63-69

19 Richards, E. W., Hamm, M. W., Fletcher, J. E. and Otto, D. A. (1990) Biochim. Biophys. Acta 1044, 361-367

20 Rose, H., Conventz, M., Fischer, Y., Jüngling, E., Hennecke, T. and Kammermeier, H. (1994) Biochem. Biophys. Acta 1215, 321-326

21 Rosendal, J., Ertbjerg, P. and Knudsen, J. (1993) Biochem. J. 290, 321-326

22 Fulceri, R., Gamberucci, A., Scott, H. M., Giunti, R., Burchell, A. and Benedetti, A. (1995) Biochem. J. 307, 391-397

23 Woldegiorgis, G., Yousufzai, S. Y. K. and Shrago, E. (1982) J. Biol. Chem. 257, 14783-14787

24 Harris, R. A., Farmer, B. and Ozawa, T. (1972) Arch. Biochem. Biophys. 150 199-209

25 Eastmond, P. E. (1998) Carbon partitioning in developing embryos of oilseed rape (Brassica napus L.) PhD thesis, University of East Anglia

26 Slabas, A. R. and Fawcett, A. (1992) Plant Mol. Biol. 19, 169-191

Received 2 November 1999/4 February 2000; accepted 8 March 2000 